\title{
Closed-Loop Control Wind Tunnel Tests on an Adaptive Wind Turbine Blade for Load Reduction
}

\author{
Thanasis Barlas *, Jan-Willem van Wingerden ${ }^{\dagger}$, Anton Hulskamp ${ }^{\ddagger}$ and Gijs van Kuik $\S$ \\ Delft University Wind Energy Research Institute, Delft, 2629 HS, The Netherlands
}

\begin{abstract}
Wind tunnel tests on a non-rotating, dynamically scaled wind turbine blade equipped with variable trailing edge geometry were carried out. The effectiveness of the system for active load reduction purposes, with the interaction between structural dynamics, aerodynamics and control was tested. The actuation of the adaptive trailing edge was based on a piezoelectric bender actuator. The full aeroservoelastic system was identified based on input and output measurement signals. A feedback controller, using strain signals on the blade root, was designed, tuned and applied on the system in order to minimize root bending moments. The results show remarkable performance in reduction of blade root strains for both open-loop and closed-loop tests. The sensitivity of various design and control parameters are analyzed both in the prescribed cases and in the feedback-controlled system.
\end{abstract}

\section{Introduction}

Reducing loads on wind turbine rotors can offer great reduction to the total cost of wind turbines. With the increasing size of wind turbine blades, the need for more sophisticated load control techniques has generated the interest for locally distributed aerodynamic control systems with built-in intelligence on the blades (often named in popular terms "smart structures" or "smart rotor control"). Recent inventory of research for such systems has been performed by Barlas and van $\mathrm{Kuik}^{4}$. Various national and European research programs deal with this subject, including the work package "Smart rotor blades and rotor control" in the Upwind EU framework program, the project "Smart dynamic control of large offshore wind turbines" sponsored by the the Dutch Technology Foundation STW and the Danish project "ADAPWING". Active load control utilizing trailing edge flaps or deformable trailing edge geometry is considered a feasible and efficient solution, especially because of advances in smart material actuator technology.

Previous work at various research groups with CFD simulations (see Troldborg ${ }^{6}$ ), 2D aeroelastic models (see Basualdo ${ }^{7}$, Buhl et $\mathrm{al}^{8}$ and Buhl et $\mathrm{al}^{9}$ ) and extended BEM models (see Andersen et $\mathrm{al}^{1}$ ), has shown the potential of applying active control through variable geometry trailing edge airfoils on wind turbine blades for load reduction purposes. Also, wind tunnel tests (see Buhl et $\mathrm{al}^{2}$ ) have quantified unsteady aerodynamic phenomena associated with prescribed excitation and control actions for certain configurations. Consequently, small scale experimental setups proving the active load control concept are needed, which take the interaction of aerodynamics and dynamics of the structure into account and show a realistic performance in reduction of structural loads. This paper describes a successful wind tunnel experiment on a dynamically scaled wind turbine blade with feedback controlled deformable trailing edge geometry, based on smart material actuation, for load reduction. The load reduction potential with a representative scaled blade model is shown and various design and control parameters are analyzed, indicating their influence in a successful "smart" blade system and providing insight in important physical phenomena. This paper presents additional information with respect to the article of van Wingerden et $\mathrm{al}^{11}$ concerning the investigated wind tunnel tests.

*PhD Student, Faculty of Aerospace Engineering, Kluyverweg 1, 2629 HS, Delft, The Netherlands.

${ }^{\dagger}$ PhD Student, Delft Center for Systems and Control, Mekelweg 2, 2628 CD, Delft, The Netherlands.

$\ddagger$ PhD Student, Faculty of Aerospace Engineering, Kluyverweg 1, 2629 HS, Delft, The Netherlands.

$\S$ Professor, Faculty of Aerospace Engineering, Kluyverweg 1, 2629 HS, Delft, The Netherlands.

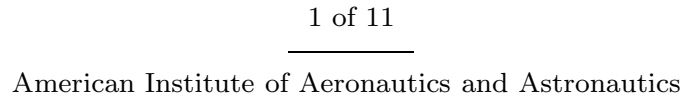




\section{Experimental Setup}

In Delft University Wind Energy Research Institute (DUWIND) an experimental wind tunnel setup was prepared. A prototype of a scaled wind turbine blade was designed with embedded load reduction control devices and feedback control was applied during experiments at the TU Delft Low Speed Low Turbulence Wind Tunnel. The design of the adaptive blade from the structural point of view is shown by Hulskamp et $\mathrm{al}^{12}$. The ultimate goal of the approach was to show that vibrations in a scaled down blade due to unexpectedly varying aerodynamic loads can be significantly reduced using trailing edge devices with an active control system. The $90 \mathrm{~cm}$ long blade model with a $12 \mathrm{~cm}$ constant chord (DU96-W-180 airfoil), constant thickness and no twist along the span was attached to a pitch system at the wind tunnel roof and it was free to deflect over a table at the free end (Fig. 1). The table ensured that no tip effects would occur that add uncertainties to measurement data. The pitch system could change the angle of attack of the blade with high speed and precision using any arbitrary signal from the control system. The characteristic length (chord) and the wind speeds used in the experiments $(30-45 \mathrm{~m} / \mathrm{s})$ correspond to Reynolds numbers in the range of $2.4 \times 10^{5}$ to $3.6 \times 10^{5}$.

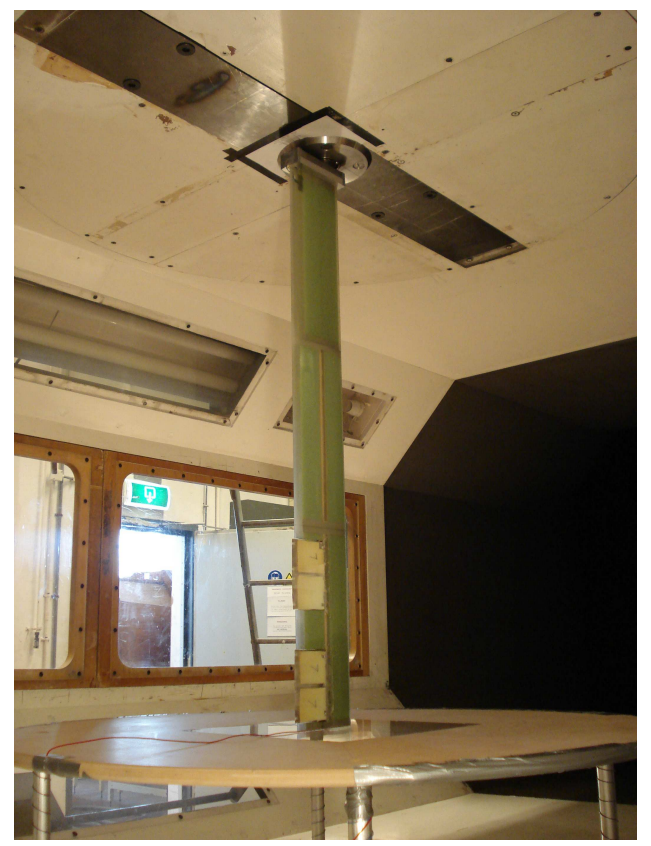

Figure 1. The adaptive blade at the TU Delft LSLT wind tunnel test section.

The glass-epoxy composite blade was designed to be representative of the dynamics of a large scale wind turbine blade. The scaling parameter used was the reduced frequency $\mathrm{k}^{\mathrm{a}}$. It was used to scale the wind field disturbance (multiples of angular frequency - 1P and 3P were considered important) as well as the first flapping natural frequency on the blade (since the devices will aim at reducing the vibrations in the out-of-plane direction). The first flapping natural frequency was tailored during the structural design of the blade (by tuning the stiffness and mass distribution). The aerodynamic excitation was simulated by the pitch excitation system. The scaled $1 \mathrm{P}, 3 \mathrm{P}$ excitations and the first flapping natural frequency of the blade were $3.5,10.5$ and $12.5 \mathrm{~Hz}$ respectively.

The aerodynamic control devices used were based on the concept of deformable trailing edge (or partial camber control). Four Thunder ${ }^{\complement}$ TH-6R piezoelectric bender actuators were used, forming two different flaps of $50 \%$ chord length and $10.5 \mathrm{~cm}$ spanwise length, placed near the tip. The thin actuators were covered with soft foam, in order to keep the trailing edge aerodynamic shape, and a latex skin, which can expand under

\footnotetext{
${ }^{\text {a}}$ The reduced frequency $k$ is defined as $k=\omega \cdot c / 2 V$, where $\omega$ is the angular frequency of the unsteadiness, $c$ is the blade's chord and $V$ is the wind speed.
} 


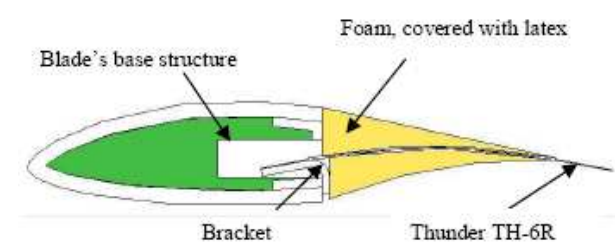

Figure 2. Design and mounting of the actuators.

the actuator deflection, providing a reasonably smooth aerodynamic surface (Fig. 2). Two piezoceramic (PZT) sensors were used in the blade root in order to measure the change in blade root bending strains. Because of the positioning of the two sensors (one far from the neutral axis for flapwise bending and one close to it, close to the trailing edge), strains both in the flapwise and edgewise directions could be measured. The piezoelectric effect at the PZT transforms deformations by elongation in two directions into voltage. In the two different positions, the strains in the flapwise or edgewise directions are dominant, although the signal is still a combination of both. Control and data acquisition were applied using a dSpace ${ }^{(C)}$ system linked to the Control Desk GUI in Matlab Simulink $`$. Through the ADC (analogue to digital converter) interface of the unit all measured signals were passed, whereas through the DAC (digital to analogue converter) interface, command signals were applied to the pitch system (to induce arbitrary disturbances) and to the flap actuators (to cancel out disturbances). This concept was used in both an open-loop and a closed loop fashion as described in the following sections.

\section{Results}

\section{III.A. Feed-forward control cases}

The main tests that have been carried out were feed forward (open loop) and feedback (closed loop) control cases. For the feed forward cases, prescribed sinusoidal motions of the pitch and the counter-acting (both) flaps for different amplitudes and frequencies were carried out. Furthermore, measurements at different mean angles of attack of interest were performed and the sensitivity of the phase angle between the two motions (pitching and flapping) was examined. The prescribed measurement cases are considered appropriate for investigating the parameters associated with the experiment

The different unsteady motions involved in this experiment are: the pitching motion of the blade introduced by the linear pitch actuator (pure harmonic variation in angle of attack), the plunging motion of the blade due to bending under unsteady aerodynamic loading, and, the trailing edge flap deflection. In the prescribed (open-loop) cases the flap is subject to a harmonic motion with the same frequency as the blade pitching, configured to act with a specific phase delay in relation to the pitching excitation.

Since the measurements are performed in a dynamic way, the unsteady motions introduce unsteady aerodynamic forces. The reduced frequency corresponding to each motion describes the unsteadiness of the aerodynamic phenomena which are crucial for any design, analysis and control of such an aeroservoelastic system. The reduced frequencies associated with the pitching motion at scaled $1 \mathrm{P}$ and $3 \mathrm{P}$ frequencies are 0.03 and 0.09 respectively (at $\mathrm{V}=45 \mathrm{~m} / \mathrm{s}$ ). The same holds for the flapping action. The plunging motion of the blade because of its bending under unsteady aerodynamic loading will have a spectrum of frequencies associated with the blade natural frequency and the frequency of excitation. For the case of 3P loading, the dominant frequency determining the aerodynamic unsteadiness will be the scaled $3 \mathrm{P}$ frequency (which is close to the natural blade frequency in the flapwise direction), so the reduced frequency will have again a value of 0.09. Also, a range of other excitation (pitching) and control (flapping) frequencies have been investigated in an open loop manner, leading to a range of reduced frequencies from 0.0084 to 0.1173 for wind speed of $45 \mathrm{~m} / \mathrm{s}$. For $0 \leq \mathrm{k} \leq 0.05$, the flow can be considered quasi-steady. For larger reduced frequencies the unsteady effects (like varying response amplitudes and phase delays) should be considered, especially for control investigations. 
For the feed-forward cases, prescribed sinusoidal motions of the pitch and the counter-acting (both) flaps for different amplitudes and frequencies were carried out. The time plot of the reference case is shown in Fig. 3. In this result the reduction in the root strain signal between the uncontrolled and the flap controlled case can be seen, which is in the order of $95 \%$. Also, the influence of the placement of the two different strain sensors can be seen. The first sensor (measuring mostly flap-wise strains) has a reduced signal in the flapped case. The excitation is a harmonic pitching motion on the blade with an amplitude of 0.5 degrees at the scaled 3P frequency $(10.5 \mathrm{~Hz})$ at angle of attack of 3 degrees at wind speed of $45 \mathrm{~m} / \mathrm{s}$. The flaps motion (same for both flaps) has been prescribed as a harmonic signal with the same frequency as the pitching, a maximum amplitude of flap deflection (amplitude of $400 \mathrm{~V}$ on the piezoelectric actuators) and a tuned phase delay. The phase delay has been set by tuning it to provide optimal load reduction. The harmonic signal of the flaps was configured to act with a phase delay of 8.5 degrees compared to the excitation. Because of the specific operating conditions (reduced frequency of 0.09) the phase delay between the excitation (pitch) and the response (aerodynamic forces) is expected to be quite small. Also the resultant plunging motion produces an additional small delay in the response. Estimations of the these phase lags can be derived by simple frequency domain potential flow aerodynamic models for a reduced frequency of 0.09 for the specific operating conditions (see Ref.10 or Ref.3). Of course the structural phase delay should be also taken into account, especially with an excitation close to the natural frequency. All these effects are added, making the estimation rather complicated, so in the experiment the optimal activation of the flap is tuned manually. This also shows the necessity for feedback control based on measured signals rather than tuning of a prescribed control excitation.
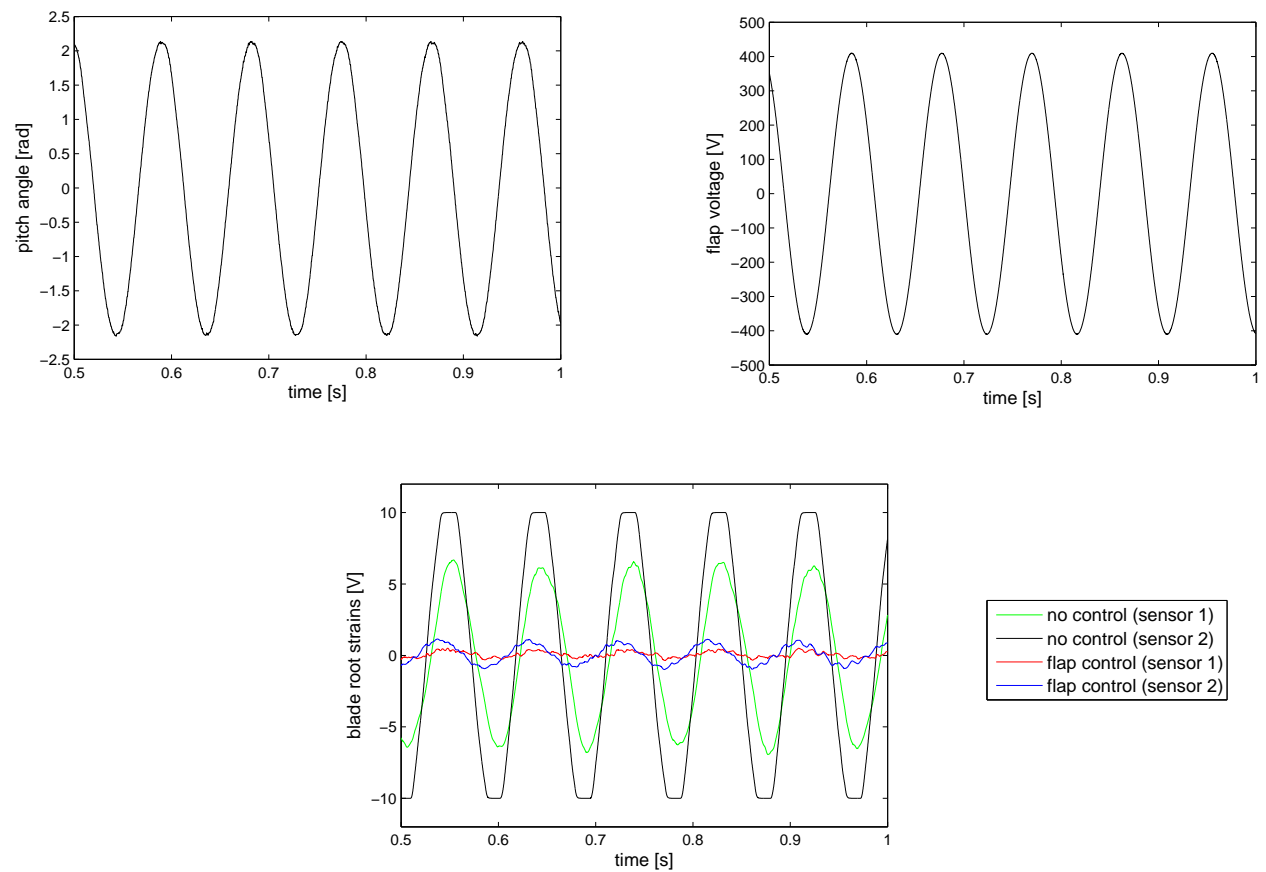

Figure 3. Reduction in root strain fluctuations in the case of a sinusoidal pitch excitation of $10.5 \mathrm{~Hz}$ with $0.5 \mathrm{degrees}$ amplitude around angle of attack of 3 degrees at $45 \mathrm{~m} / \mathrm{s}$ with prescribed counter-acting (8.5 degrees out of phase) flaps motion (feed forward control) $(1 \mathrm{~V} \simeq 21.3 \mu$ strain).

The sensitivity of various operating and control parameters has been investigated in the open-loop cases. All results are shown for the case of a scaled 3P excitation, since this is considered a more interesting case in terms of higher-harmonic control of fast fluctuations in a realistic rotor blade. The influence of the use of one or both flaps for control, and their spanwise position, in load reduction is shown in Fig. 4. It can be 
seen that the flap close to the blade tip (outboard) has a greater influence in the reduction of the blade root strains and the addition of the inboard flap doesn't affect the reduction considerably. The outboard flap has a bigger influence on the blade root strains, since it provides substantial bending moment changes because of the larger leverage. The influence of the input voltage at the flap actuators on the load reduction can be seen in Fig. 5. Maximum voltage amplitude results in maximum flap deflection (in these measurements estimated around $2 \mathrm{~mm}$ flap tip deflection which corresponds to 2 degrees flap deployment angle). The voltage range for the piezoceramic actuators, although not optimal, has been chosen to be in a normal operating range, avoiding strong non-linear effects. From the sensitivity analysis it can be seen that reducing the maximum voltage range of the piezoelectric actuators, and thus the flap deployment angle range, has a strong effect on the final load reduction performance. So optimizing the smart structure (actuator-control surface) is essential for maximum load alleviation.

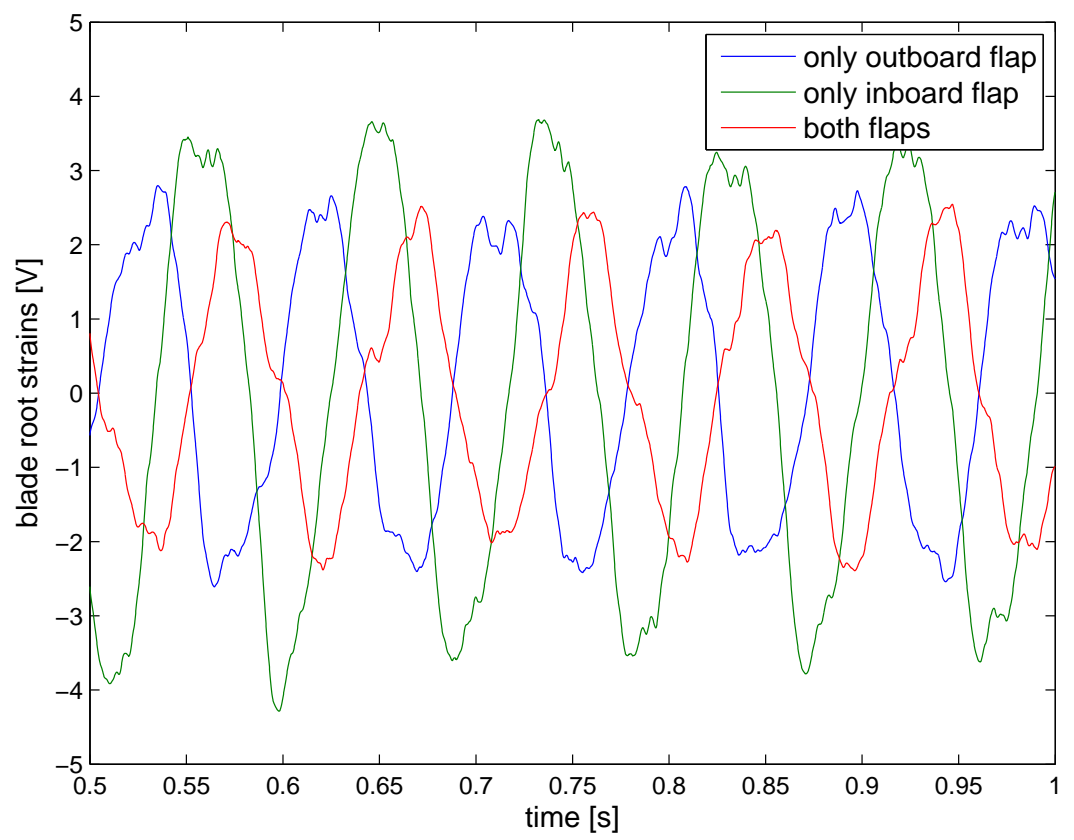

Figure 4. Sensitivity of blade root strains reduction to the number and position of flaps. Pitching $10.5 \mathrm{~Hz}$ with 0.5 degrees amplitude around angle of attack of 6 degrees at $45 \mathrm{~m} / \mathrm{s}$ with prescribed counter-acting flaps motion (feed forward control) $(1 \mathrm{~V} \simeq 21.3 \mu$ strain $)$.

The sensitivity of the phase delay between the pitching action and the flap motion is shown in Fig. 6. It is interesting to see that the maximum load reduction is achieved around a specific opposite flap phase (-0.15 rad or 8.5 degrees out of phase), when the flap action is lagging the pitching. This was explained previously considering the unsteady aerodynamic behavior at the specific operating conditions. The correct timing between the aerodynamic response due to the excitation, the structural response and the control action response determine the resultant load reduction. In a feedback control case, all of these effects are already taken into account during the control design based on system identification, so the stability and the load reduction capability of the system depend on the efficiency of the controller design and tuning.

In the wind tunnel measurements a high speed camera also had been placed at the bottom of the test section focusing on the tip of the blade. The variation of the position of the blade due to fluctuations of aerodynamic loading (from excitations and control actions) could be measured in detail from processing the captured digital camera frames. In this way the tip deflection variations have been measured. The deflections were in the range of 1 to $10 \mathrm{~mm}$ depending on the excitation frequency and the wind speed. The prescribed (opposite) control action has resulted in large reduction in both the peak value and variation of the tip deflections. Results for various test cases are summarized in Table 1. 


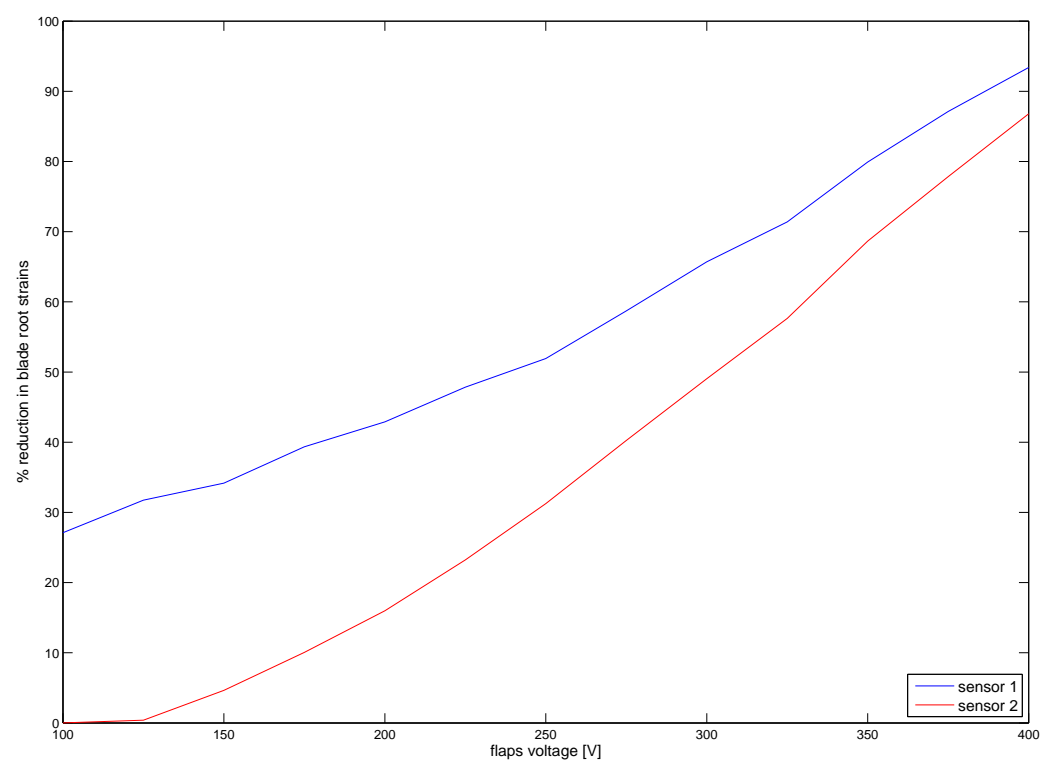

Figure 5. Sensitivity of blade root strains reduction to the voltage on piezo flap actuators. Pitching $10.5 \mathrm{~Hz}$ with 0.5 degrees amplitude around angle of attack of 6 degrees at $45 \mathrm{~m} / \mathrm{s}$ with prescribed counter-acting flaps motion (feed forward control).

Table 1. Blade tip deflections with and without feed-forward flap control at various operating conditions.

\begin{tabular}{rrrrrrr}
$\mathrm{V}[\mathrm{m} / \mathrm{s}]$ & pitch and flap freq. $[\mathrm{Hz}]$ & pitch amp. $[\mathrm{deg}]$ & $\alpha[\mathrm{deg}]$ & tip defl. $[\mathrm{mm}]$ & control & \% reduction \\
\hline 45 & 12 & 0.5 & 6 & 12.667 & off & - \\
45 & 12 & 0.5 & 6 & 0.952 & on & 92.48 \\
45 & 12.25 & 0.228 & 1 & 5.8 & off & - \\
45 & 12.25 & 0.228 & 1 & 0.7 & on & 87.9 \\
30 & 4 & 1 & 6 & 1.9 & off & - \\
30 & 4 & 1 & 6 & 0.7 & on & 63
\end{tabular}

\section{III.B. Feedback control cases}

Configuring an optimal control action for every operating condition, especially when disturbances are unknown, is not feasible. In order to efficiently reduce unsteady loads (either periodic or stochastic) a feedback control strategy must be applied. For controller design, knowledge of the dynamics of the full aeroservoelastic system is necessary. Also, a reduced order linear system is required for linear control design. Designing and applying a controller based on a model of the system (considering its complexity) is not recommended, especially with respect to system stability. In these experiments a system identification method was used in order to obtain the required knowledge of system dynamics. Specifically, the subspace system identification method was used, which uses linear algebra to estimate a state space model from measured data (see Ref. 5). The identification is done with a step signal on the pitch actuator, while a white noise signal with a bandwidth of $100 \mathrm{~Hz}$ is applied on the trailing edge flaps. The response of the system to the excitation and to the control signals is recorded from the PZT strain sensors at the blade root. The validity and performance of the subspace identification method is evaluated by the use of the Variance Accounted For (VAF) on a set of measurements different from the one used in the identification. The VAF is defined as:

$$
V A F=\max \left(1-\frac{\operatorname{var}(y-\hat{y})}{\operatorname{var}(y)}, 0\right) \cdot 100 \%
$$

\section{6 of 11}




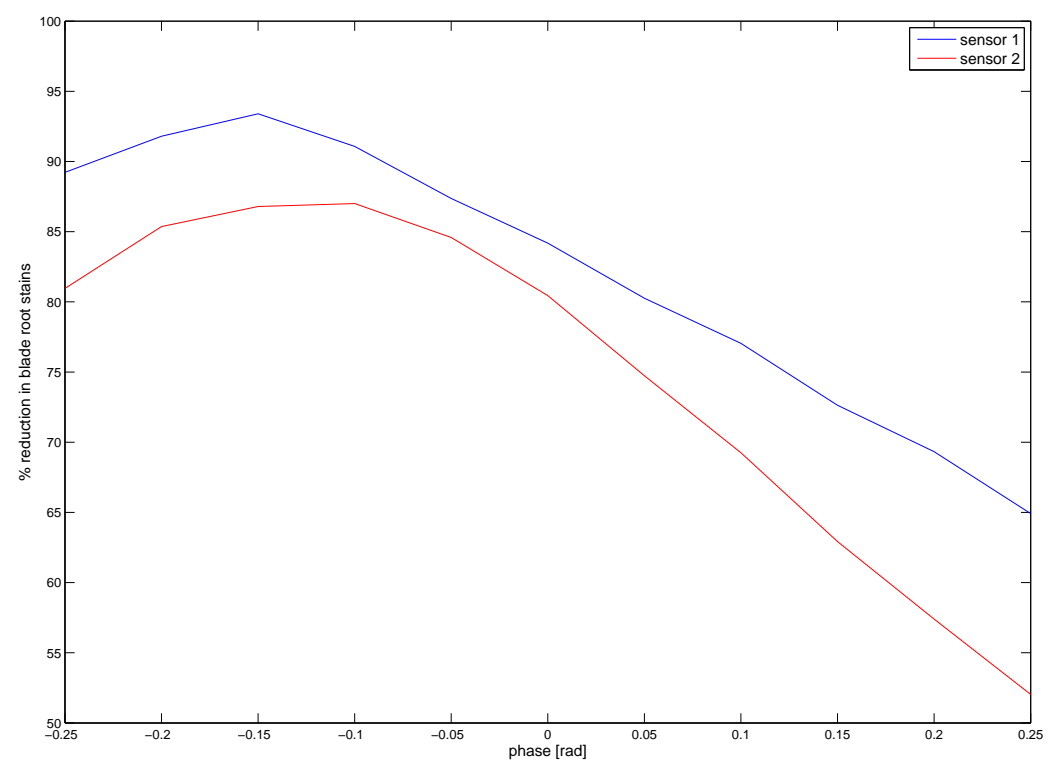

Figure 6. Sensitivity of blade root strains reduction to the phase angle between flapping and pitching action. Pitching $10.5 \mathrm{~Hz}$ with 0.5 degrees amplitude around angle of attack of 6 degrees at $45 \mathrm{~m} / \mathrm{s}$ with prescribed counter-acting flaps motion (feed forward control).

where $\hat{y}$ is the output signal of the identified state-space system, $\mathrm{y}$ is the measured signal and $\operatorname{var}()$ is the variance of a quasi-stationary signal. In Table 2 the VAF values for different wind speed and selected strain signal settings are shown.

Table 2. VAF for different wind speeds and sensor signals (ID:with identification data,VAL:validation with measured data).

\begin{tabular}{rrrrr} 
& $\mathrm{V}=30 \mathrm{~m} / \mathrm{s}$ & \multicolumn{3}{c}{$\mathrm{V}=45 \mathrm{~m} / \mathrm{s}$} \\
\hline sensor & ID & VAL & ID & VAL \\
sensor & 78.6 & 77.7 & 82.5 & 83.3 \\
& 86.9 & 86.2 & 91.7 & 91.6
\end{tabular}

In Fig. 7 the dynamic relation between the actuators and sensors is shown using a Bode plot. This plot displays the response of the system (output of strains signals) to the pitch and the flaps excitation by means of a gain and phase change in the frequency domain. Three resonance peaks can be identified, which correspond to the fist flapwise bending mode, the 1st lead-lag mode and the second flapwise mode. Also, the first sensor more easily identifies the first lead-lag mode because of its placement, even in low wind speed. The wind speed affects the amplitude and the phase of the response although does not have a big effect on the frequency of the resonance peaks. So, for efficient control design gain scheduling should be employed for different wind speeds. The angle of attack does not affect the dynamics of the system in the linear lift region (attached flow) as expected. So the identified system can be considered of the LPV form (Linear Parameter Varying) with the wind speed being the varying parameter.

From the subspace identification, a state-space model is generated. This model is appropriate for control design. A feedback controller was designed, which uses the strain signal at the blade root (voltage signal) and generates a voltage on the piezoelectric actuators, which deflect the flaps. A smoothed proportionalderivative (PD) controller with an additional notch filter was design based on the loop shaping method. An additional filter was added on the feedback signal to suppress high frequencies $(\geq 200 \mathrm{~Hz})$. Further details on the system identification and control design techniques can be found in Ref.11.

\section{7 of 11}

American Institute of Aeronautics and Astronautics 

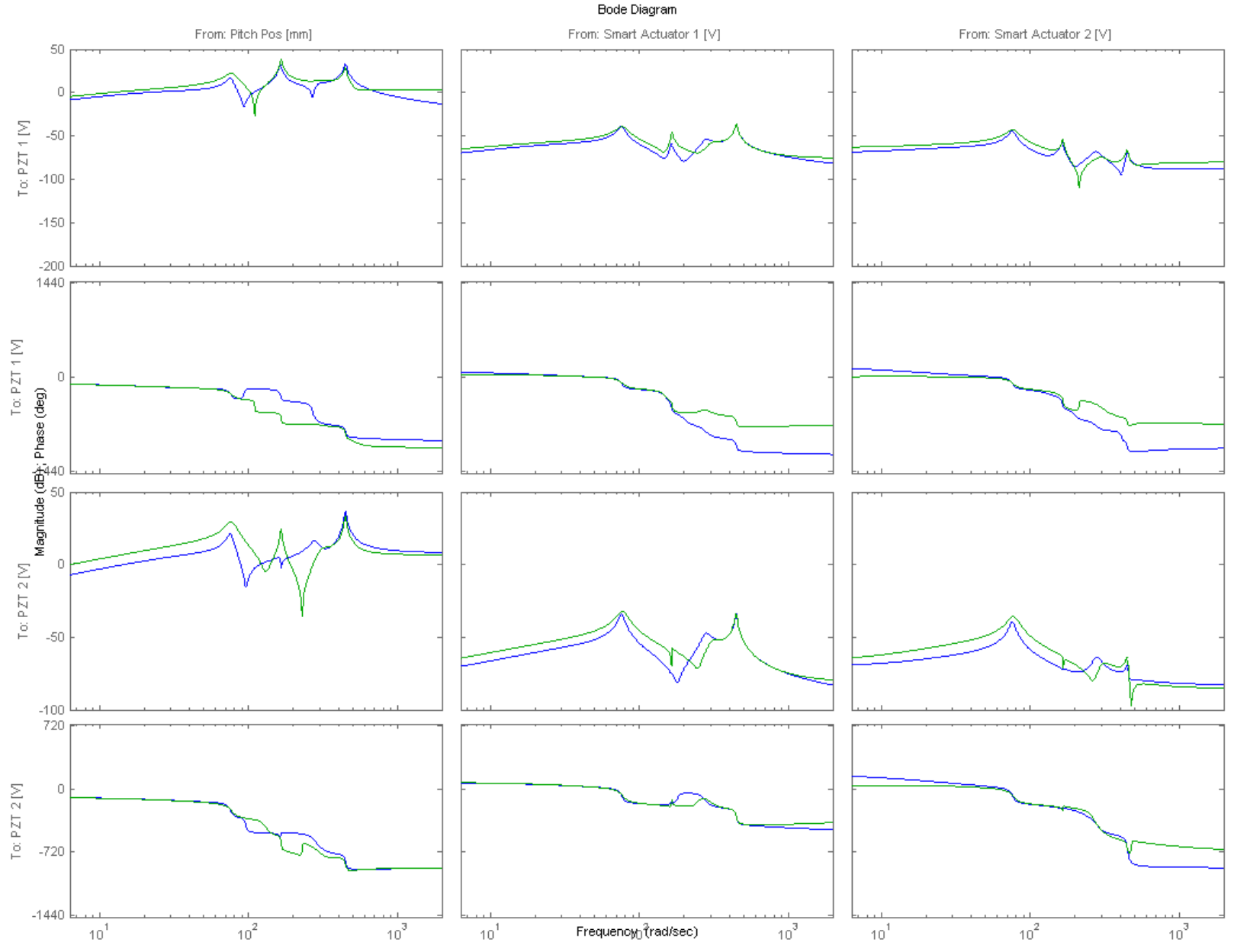

Figure 7. Bode plot of the identified system. Smart actuator 1,2: flaps, PZT 1,2: blade root strain sensors (Blue line: $\mathrm{V}=30 \mathrm{~m} / \mathrm{s}$, Green line: $\mathrm{V}=45 \mathrm{~m} / \mathrm{s})$.

This controller was initially applied to a periodic signal excitation to evaluate its performance. This test case is similar to the feed-forward control case presented previously, but now the flap motion is determined by the controller, which is effective for any operating condition (amplitude, frequency, angle of attack, but not wind speed). In the measurement results we see a reduction in the blade root strains close to $90 \%$ especially for the case of the 3P excitation. This result was expected, and it must be noted that maximum performance can not be achieved because of practical limitations (maximum control device amplitude, signal limitations, etc). The controller achieves maximum reduction around $1 \mathrm{P}$ and $3 \mathrm{P}$ excitation frequencies, although this performance can not be excepted for all frequencies. Furthermore, tip deflections were measured based on the digital camera captured frames. For case of the 3P excitation, the feedback controller reduced the tip deflection by $95.7 \%$.

The second test case for the feedback control was a step excitation at the pitch actuator. Such a signal resembles a wind gust (the worst case scenario, since the change is instantaneous). This comprises a good test for the speed of the control system actions. The results are shown in Fig. 8. The flap feedback control succeeds in damping the first flapping bending mode and reduces the peak values and the fluctuations in root strains. At high angles of attack above the static stall angle of the airfoil $\left(>8^{\circ}\right)$ we can clearly see in the blade response the addition of frequencies caused by vortex shedding because of dynamic stall. The flap is still effective and considerably reduces the increased fluctuations caused by dynamic stall. The controller is 


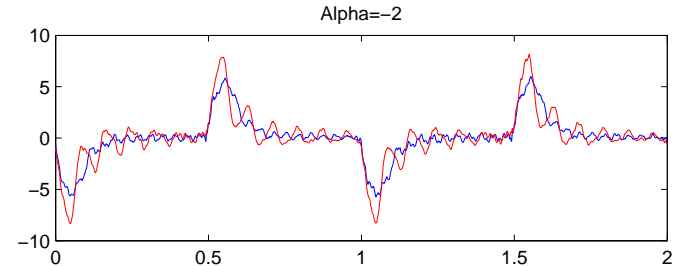

Alpha $=3$
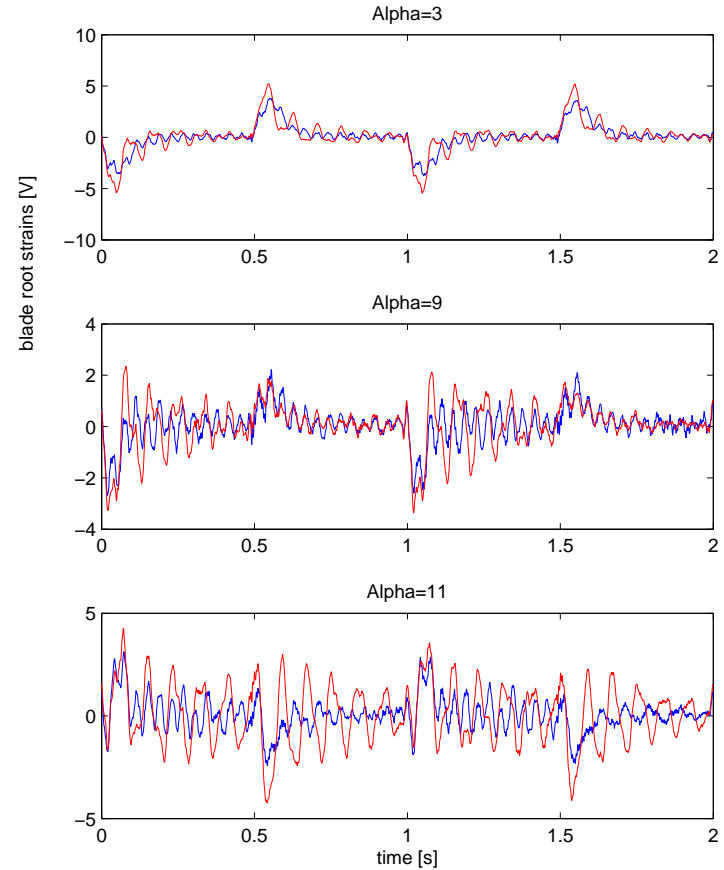

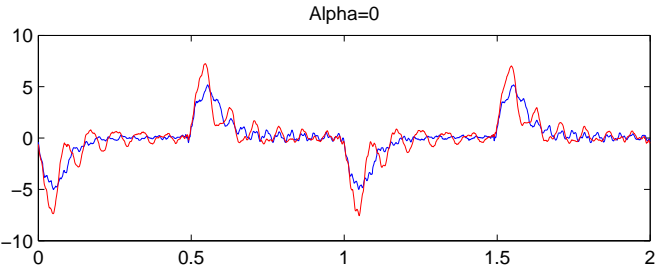

Alpha $=6$
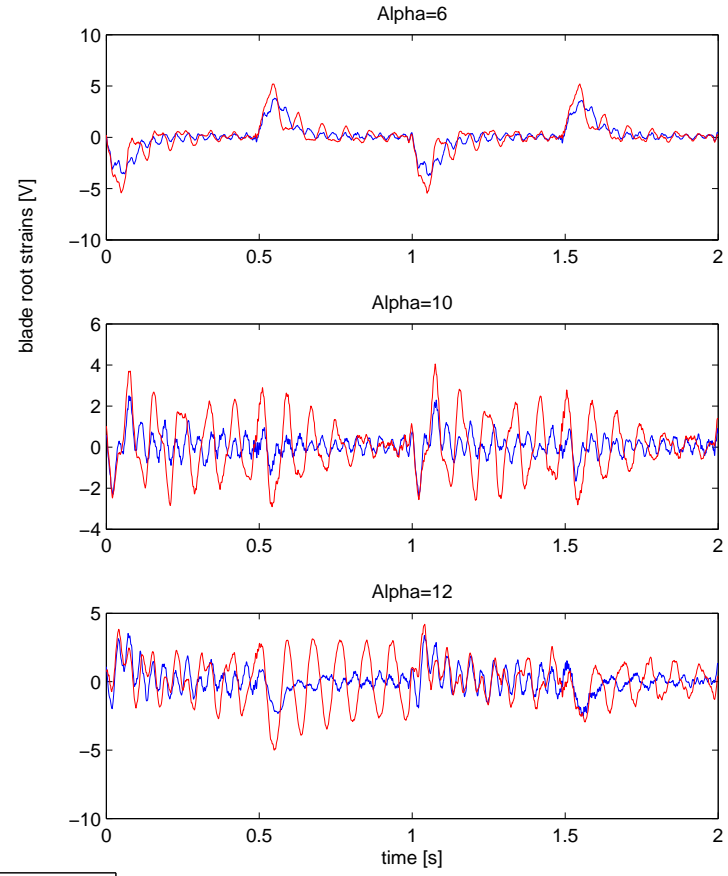

Figure 8. Feedback control on a step pitch excitation with amplitude of 1 degree at various angles of attack $(1 \mathrm{~V} \simeq 21.3 \mu$ strain).

still designed based on the system identification for smaller angles of attack. The effectiveness of the control system for such test cases proves the robustness of the controller design.

As a special test case, the aerodynamic table used to eliminate tip effects at the blade was removed. The effects induced now at the tip strongly affect the outboard flap, which is the most effective in reducing loads. The results without the table are shown in Fig. 9. The tip effects cause a reduction of loads due to smaller induced angles of attack at the tip. Also, some additional frequencies appear at the blade load response due to the 3D components of the flow, but such a case is considered hard to analyze given the type of data in these experiments.

The last but very important test case concerned feedback control on a representative stochastic signal. A filtered white noise signal was applied on the pitch actuator, simulating wind turbulence. In a real rotating blade case, the blade samples the frequency spectrum of turbulence, so the resulting variations show distinct peaks at $1 \mathrm{P}$ frequency and higher harmonics $(2 \mathrm{P}, 3 \mathrm{P})$. For that reason, the noise signal (resembling a turbulence spectrum) was filtered in order to have peaks at $1 \mathrm{P}$ and $3 \mathrm{P}$ frequencies. Since a stochastic excitation is crucial for the fatigue of the blade, the Power Spectral Density (PSD) of the input (excitation) and output (response) signals were calculated. The results show that the $3 \mathrm{P}$ frequency response of the blade is amplified more than the $1 \mathrm{P}$ because the $3 \mathrm{P}$ excitation is close to the blade first flapping frequency. With the application of the feedback controller on the flaps, the loads are considerably reduced and a reduction of

\section{9 of 11}

American Institute of Aeronautics and Astronautics 

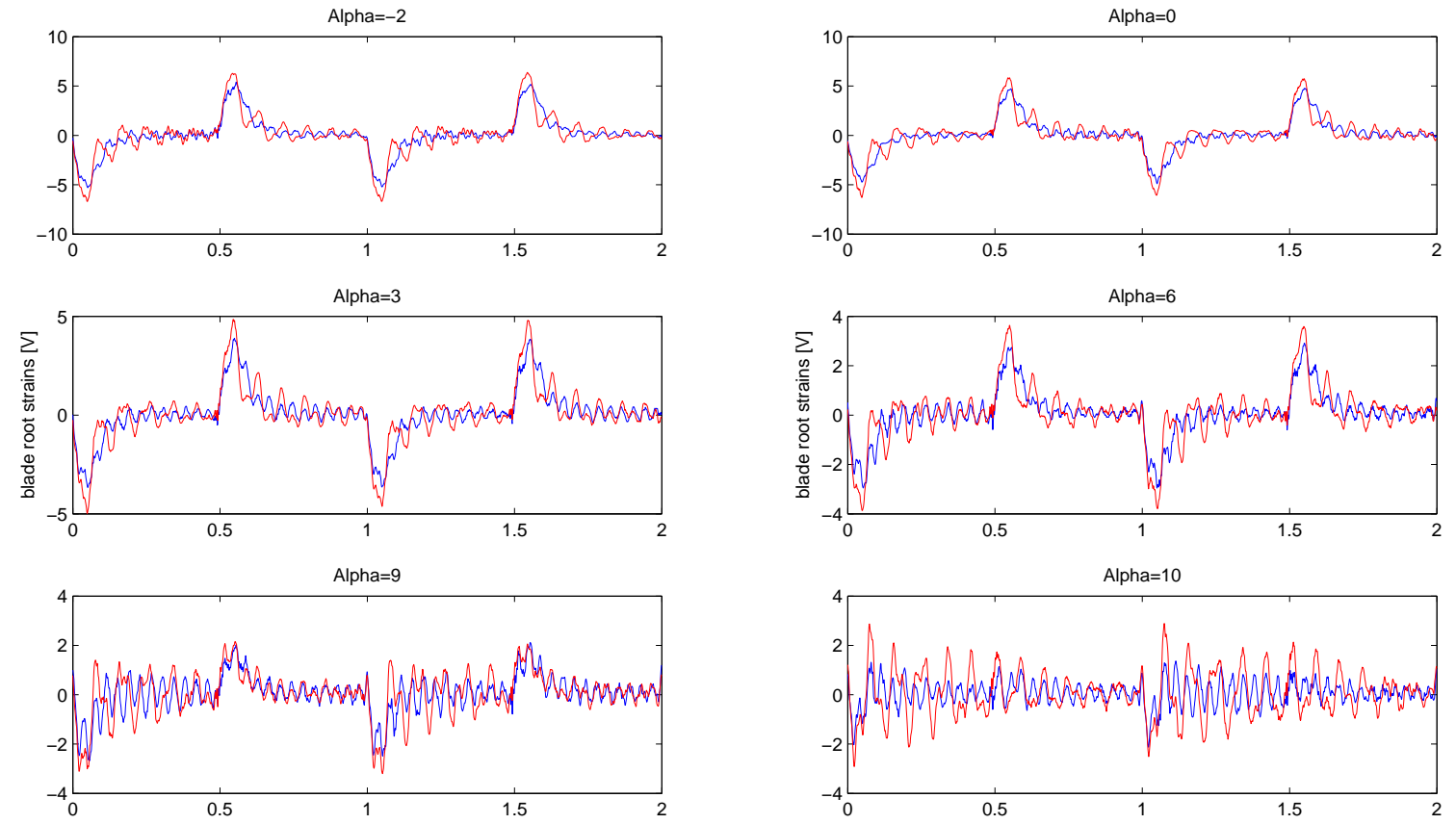

Alpha $=11$
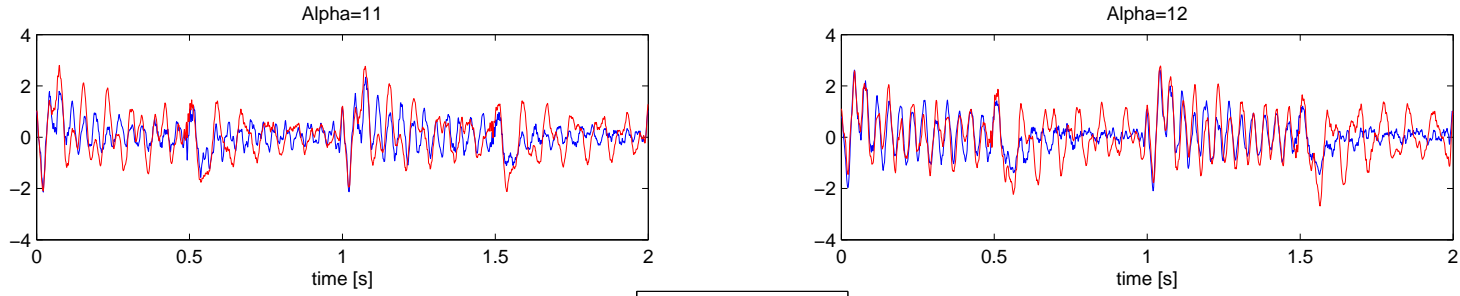

Figure 9. Feedback control on a step pitch excitation with amplitude of 1 degree at various angles of attack without the use of the aerodynamic table at the blade tip ( $1 \mathrm{~V} \simeq 21.3 \mu$ strain).

$37 \%$ and $55 \%$ in the strain standard deviation is obtained at the scaled $1 \mathrm{P}$ and $3 \mathrm{P}$ frequency respectively. More details on the feedback test cases can be found in Ref.11.

\section{Conclusions - Future work}

The application of aerodynamic control devices on a scaled wind turbine blade for active load reduction was tested in wind tunnel experiments. The prescribed control cases showed the effect of all parameters involved and demonstrated the maximum achieved load reduction. The realistic feedback control cases presented the successful application of a feedback controller based on system identification on various unsteady load cases, where the loads on the blade root were considerably reduced. Although the blade was tested in a non-rotating manner with simplified blade design, the wind tunnel test show the potential associated with the application of similar concepts on wind turbine blades for load reduction and give insight to various design and control parameters. The robustness of the controller was also proven, with significant performance on realistic simulated wind excitations.

In the future, more detailed aerodynamic measurements will take place on similar setups, identifying the unsteady aerodynamic phenomena associated with the excitations and control actions, which play a large 
role in the design of the control surfaces. Also, control tests with individually controlled flaps (spanwise distributed control) will be performed. Finally, rotating experiments will soon take place, evaluating the load reduction performance of a more realistic wind turbine rotor equipped with "smart" control devices.

\section{Acknowledgments}

The authors acknowledge the contribution of other researchers at DUWIND and technical staff who helped in the experimental setup, especially S. Toet. Smart rotor research at DUWIND in sponsored by the UpWind project, funded by the EU FP6 programme, and also partially by the Dutch Technology Foundation STW.

\section{References}

${ }^{1}$ Andersen P B, Gaunaa M, Bak C, and Buhl T. Load alleviation on wind turbine blades using variable airfoil geometry. In 2006 European Wind Energy Conference and Exhibition, 2006.

${ }^{2}$ Bak C, Gaunaa M, Andersen P B, Buhl T, Hansen P, Clemmensen K, and Moeller R. Wind tunnel test on wind turbine airfoil with adaptive trailing edge geometry. In 45th AIAA/ASME, 2007.

${ }^{3}$ Leishman J G. Unsteady lift of a flapped airfoil by indicial concepts. Journal of Aircraft, 31(2), 1994.

${ }^{4}$ Barlas T K and van Kuik G A M. State of the art and prospectives of smart rotor control for wind turbines. In The Science of Making Torque from Wind, Journal of Physics: Conference Series, 2007.

${ }^{5}$ Verhaegen $\mathrm{M}$ and Verdult V.

${ }^{6}$ Troldborg N. Computational study of the risøb1-18 airfoil with a hinged flap providing variable trailing edge geometry. Wind Engineering, 29, 2005.

${ }^{7}$ Basualdo S. Load alleviation on wind turbine blades using variable airfoil geometry. Wind Engineering, 29, 2005.

${ }^{8}$ Buhl T, Gaunaa M, and Bak C. Load reduction potential using airfoils with variable trailing edge geometry. In 43th AIAA/ASME, 2005.

${ }^{9}$ Buhl T, Gaunaa M, and Bak C. Potential load reduction using airfoils with variable trailing edge geometry. Solar Energy Engineering, 127, 2005.

${ }^{10}$ Theodorsen T. General theory of aerodynamic instability and the mechanism of flutter. Technical report, NACA, 1934.

${ }^{11}$ van Wingerden J W, Hulskamp A W, Barlas T K, Marrant B, van Kuik G A M, Molenaar D P, and Verhaegen M. On the proof of concept of a 'smart' wind turbine blade for load alleviation. Wind Energy Journal, 2008, to appear.

${ }^{12}$ Hulskamp A W, Beukers A, Bersee H, van Wingerden J W, and Barlas T K. Design of a wind tunnel scale model of an adaptive wind turbine blade for active aerodynamic load control experiments. In 16th ICCM, 2007. 